\section{Beam pump combination for fiber lasers}

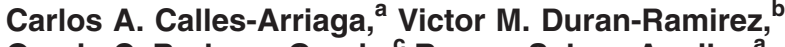

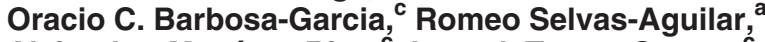 \\ Alejandro Martínez-Rios, ${ }^{c}$ Ismael Torres-Gomez, ${ }^{c}$ and \\ Ruth Mata-Chavez ${ }^{c}$ \\ auniversidad Autónoma de Nuevo Leon, Facultad de \\ Ciencias Físico-Matemáticas, CP 66450, Nuevo León, \\ Mexico \\ E-mail: charlykov@gmail.com \\ 'Universidad de Guadalajara, Centro Universitario de los \\ Lagos, CP 47460, Jal. Mexico \\ ${ }^{\mathrm{C}}$ Centro de Investigaciones en Óptica, Lomas del Bosque \\ 115, CP 37170, Gto Mexico
}

\begin{abstract}
An optical pumping device consisting of pigtailed diode lasers and a paraboloidal mirror is presented. The pigtailed diode lasers are mounted on a circular plate in radial position in front of the mirror, and the reflected beams are then launched into a $200-\mu \mathrm{m}$-diam undoped silica fiber with silicone cladding, with up to $75 \%$ coupling efficiency. The implementation of the device is simple and can be used to scale the power in fiber laser systems. (๑) 2008 Society of PhotoOptical Instrumentation Engineers.

[DOI: $10.1117 / 1.2841046]$
\end{abstract}

Subject terms: fiber lasers; combiners; high power.

Paper 070895LR received Nov. 6, 2007; revised manuscript received Dec. 13, 2007; accepted for publication Dec. 14, 2007; published online Feb. 26, 2008.

\section{Introduction}

The pumping scheme is one of the most critical elements in lasers and a particularly important issue in fiber lasers. The capability of scaling optical output power depends considerably on this element. ${ }^{1-5}$. Many techniques have been proposed so far, and these may be classified as incoherent beam combinations [also called spectral beam combining (SBC)] and wavelength spectrally coherent beam combinations (CBC) of pumping laser beams. ${ }^{6}$ Klingebiel et al. ${ }^{7}$ recently reported a SBC with combining efficiencies of $95 \%$ and with no degradation of beam quality. However, these systems are often difficult to manipulate and are also very complex. On the other hand, $\mathrm{CBC}$ techniques are usually simpler. An example consists of fiber-pigtailed pumps fused to a rare-earth-doped fiber. ${ }^{8}$ Although these pump combiners can handle high levels of power and require no alignment, the maximum power coupled is usually smaller than that reached by simple free-space end coupling. For example, a commercial $n$-pump combiner shows a typical coupling efficiency up to $90 \%$ with a maximum multimode transmission of $100 \mathrm{~W},{ }^{9}$ whereas in free-space end coupling, the launching power is many times larger than that. Notwithstanding, the latter method is limited only by the thermal fracture limit of the host material, which is typically $1150 \mathrm{~W} / \mathrm{m}$ on the facet fiber. ${ }^{10}$ In our technique, we explore a very simple spatial combination of diode lasers, in which there is no need to improve the beam quality, as there is a low coherent addition, and which permits ad-

0091-3286/2008/\$25.00 @ 2008 SPIE equate manipulation of the beams. Rather than focusing on reaching the highest possible power, the main interest of our work is to design and test both theoretically and experimentally a system that enables us to investigate the considerations to scale the optical pump power for double-clad fiber lasers with acceptable coupling efficiency. The significance of this device is that it can be easily manufactured and effectively implemented in cladding-pumped fiber lasers.

\section{Optical Design}

Figure 1 shows the scheme of the pump combiner proposed in this work. This consists of a laser system composed of two or more pumping lasers and a paraboloidal mirror. The SubMiniature version A (SMA) connectors are mounted radially in a circular plate, in whose central part is placed a commercial SMA901 connector before a focus lens. The SMA connectors mounted radially have a collimator lens included ${ }^{11}$ which can be aligned. The central SMA901 connector allows fixing a double-cladding rare-earth-doped fiber or an undoped silica fiber with silicone cladding as our current case (collector fiber). The mirror has a diameter of $100 \mathrm{~mm}$, an aperture of $65 \mathrm{~mm}$, and a focal distance of $100 \mathrm{~mm}$, with a thin golden film.

\section{Simulation of the Optical Design}

A simulation was performed using the optical design software OSLO. By computing the position of the focus of the mirror using ray traces, the real position of the central hole is found (where the collector fiber is located). This component needs to be aligned with the optical axis of the mirror. The collimated light beams from the diode lasers are adjusted in a parallel way to the optical axis of the paraboloidal mirror, the output beam of the pigtailed diodes are directed to the mirror, and these are retroreflected in a position where the silica fiber is located, as shown in Fig. 2(a). The concentrated beam then does not have spherical aberration because we assumed that the mirror is perfectly paraboloidal and compensates for that aberration. For the same reason, the off-axis aberrations like comma and astigmatism are not present. Since a mirror is used to concentrate the light, there is no chromatic aberration either. From the simulations of a previous investigation, ${ }^{12}$ we found that at distances of $1 \mathrm{~mm}$ from the mirror focus, the size of the spot is smaller than $100 \mu \mathrm{m}$, which results in certain tolerance in the defocusing of the rays toward the fiber, as shown in Fig. 2(b). Furthermore, the numerical aperture of the mirror has a value of 0.325 , which is smaller to one of

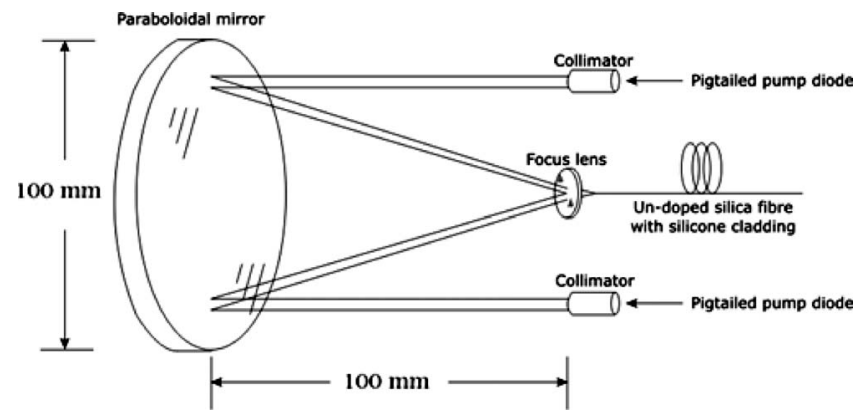

Fig. 1 Pump combiner scheme. 


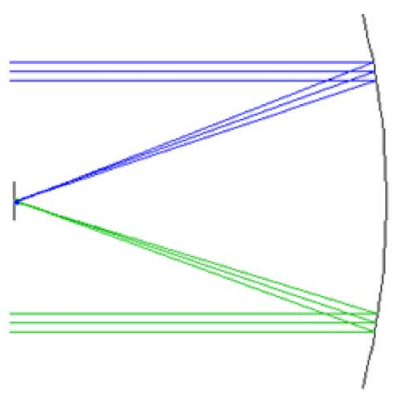

(a)

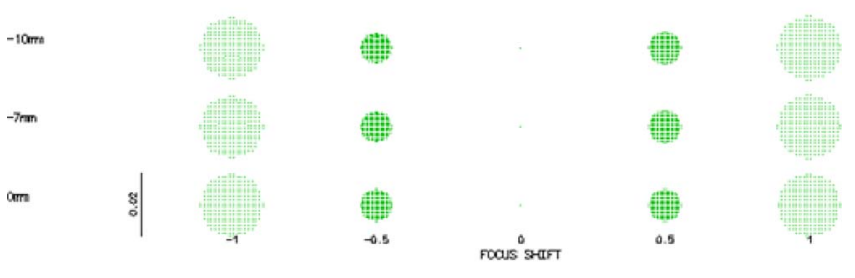

(b)

Fig. 2 Simulation of the focused beams: (a) at the mirror and (b) at the focal point.

the receiving double-clad optical fibers $(\mathrm{NA}>0.4)$, which also guarantees good coupling efficiency. In the implementation of this device, there are some features that directly affect the efficiency of the system. The first factor is related to the height of the pumping sources at the plate. We varied this parameter from 10 to $30 \mathrm{~mm}$ through simulations and we found that, unlike using a paraboloidal mirror, this does not affect the beam position at the fiber. The second case consists of the angle at which the laser diode ray reaches the paraboloidal mirror surface. Small variations of the incident ray angle significantly affect the reflected ray position. The third case is related to the tilt of the mirror itself, which affects the deviation of the focused beams from the optical axis.

\section{Experimental Setup and Results}

Figure 3 shows the experimental setup of the combiner system. We worked with three 5 -W pigtailed laser diodes at $915 \mathrm{~nm}$, with 100- $\mu \mathrm{m}$ aperture and 0.2 NA. These beams were collimated with a fiber collimator F220SMA, and all the beams retroreflected by the paraboloidal mirror were

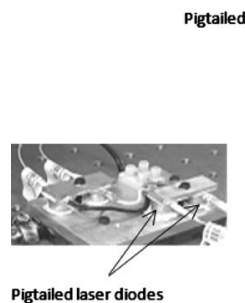

(a)

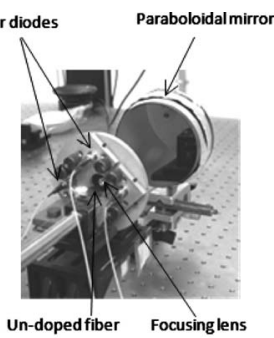

(b)

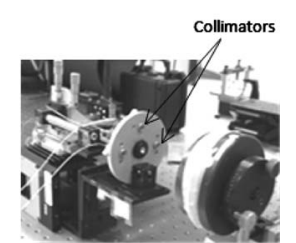

(c)
Fig. 3 Experimental combiner setup: (a) set of the pump diodes, (b) front view of the paraboloidal mirror, and (c) front view of the circular plate. focused to the collector silica fiber using a fiber collimator F240SMA from Thorlabs. ${ }^{13}$ The experimental coupling efficiency results of the undoped silica fiber were $75 \%$ for $5 \mathrm{~W}$ (one pump diode), $73 \%$ for $10 \mathrm{~W}$ (two pump diodes), and $72 \%$ for $15 \mathrm{~W}$ (three pump diodes). Two important requirements were very decisive in the selection process of such a device. The first one corresponds to the ability to scale as many diode lasers as possible. The number of pump modules can be increased as long as the dimension of the special mirror and the size of the circular plate also increases. The second factor in the design was to determine the effective numerical aperture of the special mirror, which we considered to have a value of less than 0.4 , which corresponds to the numerical aperture of our collector silicone-cladding fiber. With these parameters under control, we expected the system to work well. Moreover, an increase in the reflectivity of the mirror would improve the efficiency in our system. In our results, a decrease in the coupling efficiency was observed when the quantity of laser diodes is increased. This may be the result of absorption losses caused by the mirror and some technical problems in the adjusted mechanical screws.

\section{Conclusions}

We design, construct, and test the performance of a novel pump combiner system for cladding-pumped fiber lasers. The device consists of a simple scheme for a spatial combination of several pigtailed pump diodes and utilizes a paraboloidal mirror to couple beams into an undoped silica fiber with silicone cladding. Since the mirror is capable of handling great amounts of power, the maximum input power that can be coupled through this device is only limited by the diode lasers. Parameters such as alignment were tested both numerically and experimentally, giving results with matched values. Among the advantages of this system is its ease, in scaling the input power of a general laser system. The experimental coupling efficiency achieved in this work was 75 and $72 \%$ for one and three laser diodes, respectively, in an undoped $200-\mu \mathrm{m}$ silica fiber. This pump efficiency reduction can be attributed to the mirror absorption as well as optical misalignment; however, another alternative to increase the coupled pump power can be done by the deposition of thin films of higher reflectivity on the mirror. The performance of this pump combiner shows that it can be effectively implemented in high power systems. Additionally, if we consider the size of the paraboloidal mirror and the dimension of the circular plate that holds the SMA connectors of the $n$-number of pigtailed diode lasers, the maximum number of diodes estimated is 8 . Then, with this design, the estimated highest pump power for our coupling efficiency of $72 \%$ is roughly $28.8 \mathrm{~W}$. However, by using the same operation principle, this pump power can be increased as long as the diameter of the paraboloidal mirror and the dimension of the circular plate increase. We then conclude that the power scaling can be extrapolated with good results in practical double-clad fiber lasers.

\section{Acknowledgments}

The authors would like to express their thanks to the support of the National Council of Science and Technology (CONACyT) of Mexico for the support in the development of this work under grant J4773-F. The authors also want to 
thank Daniel May-Arrioja for his help in the deposition of the golden thin film in the mirror, and the staff of the National Institute of Astrophysics, Optics, and Electronics (INAOE) for allowing us to use some home-made paraboloidal mirrors.

\section{References}

1. P. Peterka et al., "Experimental demonstration of novel end-pumping method for double-clad fiber devices," Opt. Lett. 31(22), 3240-3242 (2006).

2. K. I. Ueda, H. Sekiguchi, and H. Kan, " $1 \mathrm{KW}$ cw output from fiber embedded laser," in Proc. Conf. Lasers Electro-Optics, postdeadline paper CPDC4 (2004).

3. L. Goldberg, J. Koplow, and D. Kliner, "High efficient 3 W side pumped Yb- fiber amplifier and laser," in Proc. Conf. Lasers ElectroOptics, pp. 11-12 (1999).

4. A. Kosterin, V. Temyanko, M. Fallahi, and M. Mansuripur, "Tapered fiber bundles for combining high-power diode lasers," Appl. Opt. 43(19), 3893-3900 (2004)

5. B. M. Flusche, T. G. Alley, T. H. Russell, and W. B. Roh, "Multi-port beam combination and cleanup in large multimode fiber using stimu- lated Raman scattering," Opt. Express 14(24), 11748-11755 (2006).

6. S. U. Alam, J. Nilsson, P. W. Turner, M. Ibsen, A. B. Grudinin, and A. Chin, "Low cost multi-port reconfigurable erbium doped cladding pumped fiber amplifier," in Proc. Euro. Conf. Opt. Commun. Germany (2000).

7. S. Klingebiel et al., "Spectral beam combining of Yb-doped fiber lasers with high efficiency," J. Opt. Soc. Am. B 24(8), 1716-1720 (2007).

8. P. Yan, M. Gong, C. Li, P. Ou, and A. Xu, "Distributed pumping multifiber series fiber laser," Opt. Express 13(7), 2699-2706 (2005).

9. OFS Catalog, High Power Products-Power combiners, see http:// www.specialtyphotonics.com/pdf/products/specialty/highpower/ 122 123.pdf.

10. L. Zenteno, "High power double-clad fiber lasers," J. Lightwave Technol. 11(9), 1435-1446 (1993).

11. Thorlabs Catalog, Fiber Optic Collimator/Coupling Packages SMA, see http://www.thorlabs.com/thorProduct.cfm?partNumber $=$ F220SMA-C".

12. R. Selvas-Aguilar, A. Martinez-Rios, and I. Torres-Gomez, "Power combiner for high-power diode lasers," Proc. SPIE 6046, 60462F (2006).

13. Thorlabs Catalog, Fiber Optic Collimator/Coupling Packages SMA, see http://www.thorlabs.com/thorProduct.cfm?partNumber =F240SMA-C". 